\title{
Zur gemeinsamen Interpretation von Haupt- und Interaktionseffekten in linearen Regressionsmodellen
}

\section{Eine kurze Gegendarstellung zum Kommentar von Reinhard Schunck und Natascha Nisic}

\author{
Jochen Mayerl · Dieter Urban
}

Online publiziert: 8. April 2020

(C) Der/die Autor(en) 2020

In Ihrem Kommentar wiederholen Schunck und Nisic eingehend den bereits in unserem Beitrag ,Vorsicht (!) bei Regressionsanalysen mit Interaktionsvariablen. Die Skalierung der unabhängigen Variablen kann die Höhe, Richtung und Signifikanz der Regressionskoeffizienten bestimmen“ (Mayerl und Urban 2019) ausführlich dargestellten Sachverhalt, dass Regressionskoeffizienten in Interaktionsmodellen als konditionale Effekte zu interpretieren sind. Mithin entspricht die Kritik von Schunck und Nisic, nach der wir vorschlagen würden, Haupt- und Interaktionseffekte isoliert voneinander zu interpretieren, nicht der Aussage unseres Beitrags. Vielmehr haben wir ausführlich ausgearbeitet, dass die isolierte Interpretation von Haupteffekten in Interaktionsmodellen hochgradig irreführend sein kann.

Eine längere Fassung dieser Gegendarstellung ist online unter www.kzfss.unikoeln.de/sites/kzfss/pdf/Mayerl.pdf einsehbar.

Funding Open Access funding provided by Projekt DEAL.

Open Access Dieser Artikel wird unter der Creative Commons Namensnennung 4.0 International Lizenz veröffentlicht, welche die Nutzung, Vervielfältigung, Bearbeitung, Verbreitung und Wiedergabe in jeglichem Medium und Format erlaubt, sofern Sie den/die ursprünglichen Autor(en) und die Quelle ordnungsgemäß nennen, einen Link zur Creative Commons Lizenz beifügen und angeben, ob Änderungen vorgenommen wurden.

\footnotetext{
J. Mayerl $(\bowtie)$

Fakultät für Human- und Sozialwissenschaften, Technische Universität Chemnitz

Thüringer Weg 9, 09126 Chemnitz, Deutschland

E-Mail: Jochen.Mayerl@soziologie.tu-chemnitz.de

D. Urban

Institut für Sozialwissenschaften, Universität Stuttgart

Seidenstraße 36, 70174 Stuttgart, Deutschland

E-Mail: dieter.urban@sowi.uni-stuttgart.de
} 
Die in diesem Artikel enthaltenen Bilder und sonstiges Drittmaterial unterliegen ebenfalls der genannten Creative Commons Lizenz, sofern sich aus der Abbildungslegende nichts anderes ergibt. Sofern das betreffende Material nicht unter der genannten Creative Commons Lizenz steht und die betreffende Handlung nicht nach gesetzlichen Vorschriften erlaubt ist, ist für die oben aufgeführten Weiterverwendungen des Materials die Einwilligung des jeweiligen Rechteinhabers einzuholen.

Weitere Details zur Lizenz entnehmen Sie bitte der Lizenzinformation auf http://creativecommons.org/ licenses/by/4.0/deed.de.

\section{Literatur}

Mayerl, Jochen, und Dieter Urban. 2019. Vorsicht (!) bei Regressionsanalysen mit Interaktionsvariablen. Kölner Zeitschrift für Soziologie und Sozialpsychologie 71: 135-156.

Jochen Mayerl Prof. Dr., Professor für Soziologie mit Schwerpunkt Empirische Sozialforschung am Institut für Soziologie der Technischen Universität Chemnitz. Forschungsschwerpunkte: Moderatoren und Mediatoren der Einstellungs-Verhaltens-Relation, Umfrageforschung und Strukturgleichungsmodellierung. Aktuelle Publikationen: Responding to socially desirable and undesirable topics: Different types of response behavior? Methods, Data, Analyses 2018 (m. H. Andersen); Two worlds of environmentalism? Empirical analyses on the complex relationship between Post-Materialism, National Wealth and Environmental Concern. Nature + Culture 13, 2018 (m. H. Best); Attitudes towards Muslims and fear of terrorism. Ethnic and Racial Studies 41, 2018 (m. H. Andersen)

Dieter Urban Prof. Dr., Univ.-Professor für Soziologie am Institut für Sozialwissenschaften der Universität Stuttgart. Forschungsschwerpunkte: Theoretische und statistische Modellierung von sozialen Strukturen und Prozessen. Aktuelle Buchpublikation: Angewandte Regressionsanalyse: Theorie, Technik und Praxis. Wiesbaden 2018 (zus. mit J. Mayerl). 